\title{
Electrochemical behaviour and voltammetric sensitivity at arrays of nanoscale interfaces between immiscible liquids
}

Mickaël Rimboud, ${ }^{a}$ Robert D. Hart, ${ }^{b}$ Thomas Becker ${ }^{a}$ and Damien W. M. Arrigan ${ }^{a, *}$

\footnotetext{
${ }^{a}$ Nanochemistry Research Institute, Department of Chemistry, Curtin University, GPO Box U1987, Perth, WA 6845, Australia.
}

${ }^{\mathrm{b}}$ Centre for Materials Research, Department of Imaging and Applied Physics, Curtin University, GPO Box U1987, Perth, WA 6845, Australia.

*Corresponding author. Email d.arrigan@curtin.edu.au; fax +61-8-9266-2300. :

\begin{abstract}
Arrays of nanoscale interfaces between immiscible electrolyte solutions were formed using silicon nitride nanopore array membranes. Nanopores in the range from $75 \mathrm{~nm}$ radius down to $17 \mathrm{~nm}$ radius were used to form the nano-interfaces. It was found that the liquid organic phase electrolyte solution filled the pores so that inlaid nano-interfaces were formed with the aqueous phase. Cyclic voltammetry at these nano-interface arrays demonstrated steady-state behaviour at the larger interfaces but the voltammetric wave-shape became progressively worse as the interface size decreased. It was found that the ion transfer currents were ca. $50 \%$ of those expected based on theoretical calculations, which is attributed to overlap of diffusion zones at adjacent nanointerfaces. Here, the separation between adjacent nano-interfaces was 20-times the interface radius. The analytical sensitivity for ion transfer from the aqueous to the 1,6-dichlorohexane organic phase was estimated from calibration plots of current density versus concentration of
\end{abstract}


aqueous tetraethylammonium cation. The sensitivity was in the range of $65 \mu \mathrm{A} \mathrm{cm}{ }^{-2} \mu M^{-1}$ (at 75 $\mathrm{nm}$ radius interfaces) to $265 \mu \mathrm{A} \mathrm{cm}{ }^{-2} \mu \mathrm{M}^{-1}$ (at $17 \mathrm{~nm}$ radius interfaces). The sensitivity depended directly on the inverse of the nano-interface radius, implying that smaller interfaces will provide better sensitivity, due to the enhanced flux of analyte arising from convergent diffusion to smaller electrochemical interfaces.

\section{Introduction}

Electrochemistry at the interface between two immiscible electrolyte solutions (ITIES) enables the study of both electron- and ion-transfer processes, ${ }^{1,2}$ thus providing a platform for the sensing and determination of redox inactive ionised species $^{3}$. A major trend in recent years in this branch of electrochemistry has been the development and study of processes at smaller and smaller interfaces, down to the microscale and the nanoscale ${ }^{4}$. The purpose of this miniaturisation is, as for solid electrodes, ${ }^{5,6}$ to gain an enhanced mass transport flux, providing greater current densities and therefore a higher sensitivity of the analytical response ${ }^{7,8}$. Moreover, the decrease of the interface dimension leads to a lowering of the interfacial capacitance and hence background charging current. The low currents recorded at such small interfaces has permitted studies in resistive media ${ }^{9,10}$ or in the presence of low concentrations or even absence of deliberately-added supporting electrolyte ${ }^{11}$.

Two approaches have been widely used to establish nanoscale miniaturized ITIES. In the first

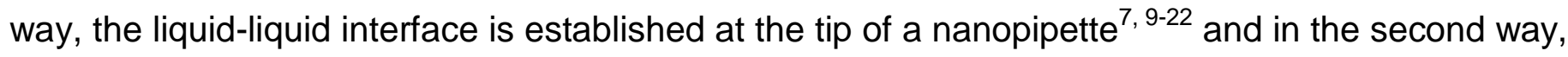
the interface is established within a nanoporous material ${ }^{23-25}$. Most of the work published to-date on liquid-liquid nano-interfaces has been performed with the nanopipette-based approach. This method allows the formation of one or two (in the case of double-barrelled nanopipettes ${ }^{9,15,19}$ ) liquid-liquid nano-interface(s). The study of the kinetics of ion transfer at such nano-interfaces has been the focus of study, and this approach was recently extended to study ion transfer kinetics at 
a nano-interface between water and an ionic liquid ${ }^{22}$. In contrast to the nanopipettes, nanoporous materials allow the formation of numerous nano-interfaces within a defined area. An important point in the design of these nanoporous material-supported multiple interfaces is to control the geometric parameters of the pore array, namely the pore radius and the pore-to-pore separation. This can be achieved by the use of nanofabrication technologies employed in semiconductor device fabrication. Previously, the fabrication of nanopore arrays in silicon nitride membranes by combination of electron-beam lithographic (EBL) patterning and reactive ion etching (RIE) was reported $^{26,27}$. In such an approach, the geometric parameters were well-controlled and the arrays were close to the designed patterns. These nanopore arrays were then used in voltammetric experiments at water | 1,6-dichlorohexane (gellified) interfaces ${ }^{26,}{ }^{28}$. Electrochemical characterisation of the nano-interface arrays was carried out by cyclic voltammetry of tetraethylammonium cation transfer. In particular it was found that the current density for ion transfer voltammetry increased with decreasing pore size (in the range of pore radius $25-230$ $\mathrm{nm})^{26}$. It was also found that the sensitivity (slope of the calibration plot) increased by two orders of magnitude on miniaturising the interfaces from millimetre-sized through micrometre-sized to nanometre-sized interfaces ${ }^{28}$. The sensitivity at the array of smallest interfaces examined (radius $70 \mathrm{~nm})$ was $63 \mu \mathrm{A} \mathrm{cm}{ }^{-2} \mu \mathrm{M}^{-128}$.

In this report, we present further characterisation studies of the behaviour of nano-interface arrays formed at silicon nitride nanoporous membranes. The emphasis of this work was examination of interface size across the nanometre range and the impact of this on sensitivity. Previously, the dependence of analytical sensitivity on interface size compared only one nanoscale interface size (ca. $70 \mathrm{~nm}$ radius) with a micrometre interface array and a millimetre-sized interface ${ }^{28}$. The results presented here demonstrate that sensitivity does increase as nano-interfaces of decreasing size are employed. However it is also apparent that measurement precision becomes worse with decreasing nano-interface size. 


\section{Experimental}

\section{Reagents and materials}

All reagents used were purchased from Sigma-Aldrich Australia and used without further purification unless indicated otherwise here. Deionised water was obtained from a Milli-Q water purification system (Millipore Pty Ltd, North Ryde, NSW, Australia). The water produced by this unit had a resistivity of $18 \mathrm{M} \Omega \mathrm{cm}$. The solvent employed for the organic phase was 1,6dichlorohexane $(\mathrm{DCH})$. It was rinsed three times with purified water prior to use. Both the water and $\mathrm{DCH}$ were mutually saturated before further use. The supporting electrolytes were $\mathrm{LiCl}$ in the aqueous phase and bis(triphenylphosphoranylidene)ammonium tetrakis(4-chlorophenyl)borate (BTTPATPBCl) in $\mathrm{DCH}$, both at a concentration of $10 \mathrm{mM}$. The latter salt was prepared by metathesis from bis(triphenylphosphoranylidene)ammonium chloride (BTTPACI) and potassium tetrakis(4-chlorophenyl)borate $(\mathrm{KTPBCl})$, following the published procedure ${ }^{29}$. The model analyte used was the tetraethylammonium cation $\left(\mathrm{TEA}^{+}\right)$in its chloride form, dissolved in the aqueous phase.

The silicon nitride nanopore array membranes used to form the nano-interfaces were prepared as described previously ${ }^{26}$, using combinations of photo- and electron-beam lithography, etching and deposition.

\section{Imaging}

Scanning electron microscopy (SEM) images were recorded using a Zeiss Neon 40 EsB FIBSEM microscope (Carl Zeiss Nano Technology Systems). Images were taken with a beam of $5 \mathrm{kV}$, using the In Lens secondary electron detector.

Atomic force microscopy (AFM) images were recorded with a Dimension 3100 (Nanoman) AFM with $\mathrm{x}$-y-closed loop scanner and Nanoscope IV controller (Veeco, Santa Barbara, USA). All 
images were taken in Tapping Mode operation using Silicon Tapping Mode probes of type $\mathrm{NCH}$ (resonance frequency: $\approx 320 \mathrm{kHz}$, spring constant: $42 \mathrm{~N} \mathrm{~m}^{-1}$, (NanoWorld $\mathrm{AG}$, Neuchâtel, Switzerland).

\section{Experimental procedure}

Voltammetric experiments at nanopore-supported liquid-liquid interface arrays were performed using an Autolab PGSTAT302N (Metrohm, The Netherlands) together with Nova 1.6 software. A two electrode electrochemical cell was used, with both electrodes serving as reference and counter electrode in either phase. This was possible because of the low currents measured (nA). The organic phase $(200 \mu \mathrm{L})$ was contained in a glass tube $(2.5 \mathrm{~mm}$ inner and $4 \mathrm{~mm}$ outer diameter) with the silicon chip (5 $\mathrm{mm} \times 5 \mathrm{~mm}$ ) containing the nanopore array membrane sealed to one end with silicone sealant. This was then immersed in the aqueous phase contained in a $10 \mathrm{~mL}$ beaker. The experiments were performed with liquid $\mathrm{DCH}$ as the organic phase in contrast to the PVC-gellified organic phase used previously ${ }^{26,28}$. The electrode placed in the organic phase was a silver wire, so that it was a pseudo-reference electrode. The electrochemical cell can be summarized as follows:

\section{Ag | AgCl | LiCl (0.01 M) W | BTTPATPBCl (0.01 M) DCH | Ag}

Once the cell was set up, a background voltammogram was run over a wide potential range in order to establish the available potential window; this shifted slightly with each experiment because a pseudo-reference electrode was used. Then, a sequence of five "blank" (background) voltammograms was recorded in a potential range large enough to encompass the transfer of $\mathrm{TEA}^{+}$when it was be added to the aqueous phase. Aliquots of a $100 \mu \mathrm{M}$ TEACl solution in $0.01 \mathrm{M}$ $\mathrm{LiCl}$ in $\mathrm{DCH}$-saturated water were then added to the aqueous phase with a micropipet in order to produce the desired concentration of $\mathrm{TEA}^{+}$in the aqueous phase. All voltammetric experiments 
were performed in a Faraday cage. As a pseudo-reference electrode was used, the limiting currents were measured at $200 \mathrm{mV}$ positive of the foot of the transfer wave.

\section{Results and discussion}

\section{Nanopore array characterization by SEM and AFM}

The nanopore membranes were fabricated in silicon nitride $\left(\mathrm{Si}_{3} \mathrm{~N}_{4}\right)$ using combinations of photoand electron-beam lithography, etching and deposition ${ }^{26}$. The process results in silicon chips (5 $\mathrm{mm} \times 5 \mathrm{~mm}$ ) containing the $100 \mathrm{~nm}$ thick silicon nitride membrane supported on a $525 \mu \mathrm{m}$ thick silicon frame. The nanopores arrays are situated in a $500 \mu \mathrm{m} \times 500 \mu \mathrm{m}$ central zone of the membrane.

Arrays of 400 pores, 20 rows of 20 pores arranged in a hexagonal pattern, featuring five different radii but always the same ratio between the pore-pore separation, $r_{c}$, and the radius, $r_{a}$, were designed and characterized by SEM and AFM. It was seen previously that a large ratio $r_{\mathrm{c}} / \mathrm{r}_{\mathrm{a}}$ minimises the overlaps between diffusion zones formed at adjacent interfaces in electrochemical experiments $^{26}$. It has been shown that no overlap occurs between micro-interfaces for ratios $r_{c} / r_{a}$

of 20 or more ${ }^{30}$ hence that ratio was used in the current designs of nanopore arrays. As increased analytical sensitivity is expected with interfaces formed at smaller pores, arrays were designed with pore radii smaller than in previous work $^{26,28}$, down to $15 \mathrm{~nm}$. Selected SEM images of the nanopore arrays are shown in Figure 1 and the different geometric parameters of the arrays, as determined from the SEM images, are listed in Table 1. All arrays were in good agreement with their designs, expect for design 5, which had a design radius of $15 \mathrm{~nm}$. The slight deviation between the design and actual radius for this design is because the EBL patterning method is close to the limits of its operability. The preparation of arrays of smaller nanopores than achieved here will require use of alternative fabrication methods. The lighter rectangular areas that appeared on the images in Figure 1 are due to the exposure of these areas to the electron beam 
in previous SEM experiments. The white halo around the pores on images C and D (Figure 1) are due to edge highlighting effects from the escape of the secondary electrons in the vicinity of the pore borders. As observed, the desired pattern was reproduced in the silicon nitride membrane with high fidelity. For each design of nanopore array, the expected ratio $r_{c} / r_{a}$ of 20 is observed. This ratio is slightly smaller for design 5 as the pore radius is bigger than expected, $17 \mathrm{~nm}$ instead of $15 \mathrm{~nm}$ (Table 1). Nevertheless, this design contains smaller nanopores than in previous work, where a radius of $25 \mathrm{~nm}$ was the smallest investigated. ${ }^{26}$ Selected AFM images of the nanopore array design 5 are shown in Figure 2. The hexagonal layout of the nanopore array is clearly shown. These AFM images confirmed the pore-to-pore separation determined from SEM. Measurements of nanopore radii, however, were less accurate than those from SEM images as the extracted profiles (not shown) did not clearly delineate the pore edges. This is a result of the geometric convolution due to the AFM probe geometry, which leads to a measured pore radius which is smaller than the actual pore radius.

\section{$\mathrm{TEA}^{+}$transfer at nanopore array-supported W-DCH interface}

Ion transfer across nanopore-supported W-DCH interface arrays was characterised using cyclic voltammetry (CVs) with $\mathrm{TEA}^{+}$as the model analyte for these studies. In order to obtain the calibration curves linking limiting currents and ion concentrations, CVs were recorded at seven different concentrations of TEACl in the aqueous phase, from 20 to $140 \mu \mathrm{M}$ in steps of $20 \mu \mathrm{M}$. Before adding the analyte, a CV of the background electrolyte solutions (i.e. in the absence of TEACI in the aqueous phase) was recorded. This background CV was subsequently subtracted from those obtained in presence of $\mathrm{TEA}^{+}$to provide background-subtracted voltammograms. Recordings obtained with $10^{-4} \mathrm{M}$ of $\mathrm{TEA}^{+}$in the aqueous phase and the corresponding background-subtracted curves for each pattern of pore array studied are shown in Figure 3. 
Voltammograms show a steady-state behaviour on the forward scan, while the backward scan evolves from a steady-state with nanopore design 1 (Figure 3 A), to a peak-shaped voltammogram as the pore size decreased (Figure $3 \mathrm{C}, \mathrm{E}, \mathrm{G}, \mathrm{I}$ ). As can be observed, no true limiting current plateau is reached in the diffusion-limited region, and CVs show a steady increase of the current instead. This increasing current becomes more substantial as the nanopore size decreases. With design 5, which possessed the smallest size of pore examined, it can be seen that the analyte ion transfer wave cannot be clearly distinguished from the rising current response (Figure $3 \mathrm{I}$ and $\mathrm{J}$ ). This may be due to a reversible expansion of the interface as the ion is transferring ${ }^{31}$ and by the influence of the transfer of the supporting electrolytes ions as higher potentials are reached. It was also noted that in our experiments, the current rose more quickly with the applied potential than observed previously at the aqueous-gelified $\mathrm{DCH}$ interface. ${ }^{26}$ This may be a result of the lower viscosity of the liquid organic medium employed here. The increased current which makes the ion transfer current difficult to identify may also be attributable to leakage currents, as the voltammograms are like those with deliberately added current leakages for microelectrode random arrays $^{32}$. However, in the present case, the increased current in the diffusion-limited region seems to happen only after the transfer of an ion is observed, rather than across the whole voltammogram.

Some of the CVs, especially at the smaller pore sizes, start to show a current peak on the reverse scan. These peaks correspond to the back-transfer of TEA+from DCH to the aqueous phase and are characteristic of the influence of linear diffusion. This supports the suggestion that the nanopores are filled with the organic phase, so that the liquid-liquid interfaces are close to or coplanar with the aqueous side of the silicon nitride membrane. The interface could thus be likened to an inlaid disc electrode for the transfer of ions from the aqueous phase to the $\mathrm{DCH}$ phase. Previously it was found that peak-shaped reverse scan voltammograms were obtained after background-subtraction at the aqueous-organogel nano-interface $\operatorname{array}^{26}$. The peak shape of the reverse scan became more pronounced as the scan rate was increased, leading to the suggestion that the organogel phase filled the pores so that inlaid interfaces were formed on the aqueous side 
of the membrane ${ }^{26}$. However, in the present work, in which a liquid organic phase was employed, it seems that the electrochemical behaviour of these reverse-scan peaks is more complex than the simple result of TEA ${ }^{+}$diffusion inside the pores, as illustrated by the voltammograms in Figure 4.

As can be seen, the reverse peak intensity seems to depend on the intensity of current across the interface, i.e. on the choice of the switching potential (Figure 4A). When the switching potential is just above the transfer wave for $\mathrm{TEA}^{+}$, as exemplified by the plain line voltammogram in Figure 4A, no peak is observed on the reverse scan. But, as the switching potential is shifted gradually to higher values, the peak appears and increases (voltammograms indicated by dashed and dotted lines, Figure 4A). The behaviour of the voltammogram with the highest switching potential of the experiment is intriguing (Figure 4A, dotted line voltammogram). The reverse scan in particular shows a diffusion current higher than the forward scan, a cross-over of current between the scans and an enhanced peak compared to the situation with lower switching potentials. This behaviour, especially the cross-over producing a characteristic nucleation loop (see insets in Fig. 4A), is reminiscent of the occurrence of electrodeposition at solid electrodes ${ }^{33,34}$ and at liquid-liquid interfaces ${ }^{35,36}$. As the organic phase supporting electrolyte anion (i.e. $\mathrm{TPBCl}^{-}$) transfers in the potential region of the switching potential, an association between EEA $^{+}$and this anion could occur. This would not be surprising as ion pairing was shown to have a great influence on ion transfer at $\mathrm{W} \mid \mathrm{DCH}$ interface ${ }^{37,38}$. The ion-pairing between $\mathrm{TEA}^{+}$and $\mathrm{TPBCl}^{-}$at the polarised interface could lead to nucleation of an insoluble product at the interface, producing the characteristic voltammograms recorded. Indeed, as one of the anticipated benefits of nanointerfaces is the enhanced flux of analyte by radial diffusion, the greater flux of $\mathrm{TEA}^{+}$to the nanoITIES as the interface is made smaller may lead to enhanced nucleation of an ion-pair precipitate at the interface at the upper extremity of the potential window. This is consistent with the observed reverse peak currents increasing in magnitude as interface size decreases. The reverse peak current may thus be due to electro-dissolution of the nucleated material from the interface during the reverse scan, leading to the peak-shaped response to the stripping of material from the interface. For the dotted line voltammogram in Figure 4A, integration of the current over time of the 
forward scan after background-subtraction gives a transferred charge of $8.24 \times 10^{-9} \mathrm{C}$, which corresponds to a quantity of $8.54 \times 10^{-14}$ mol. Taking into account the volume of the nanopore array, $3.63 \times 10^{-17} \mathrm{~L}$, and assuming that all the transferred ions remain confined within the pores, it produces a concentration of $2.3 \times 10^{3} \mathrm{M}$ in the organic phase following the forward transfer process (for comparison, if this material is distributed throughout the organic phase volume, ca. $300 \mu \mathrm{L}$, the corresponding concentration is ca. $10^{-5} \mathrm{M}$ ). This is beyond all solubility limits and supports the view that the reverse peak is simply stripping of such insoluble material back into the aqueous phase. By integrating the reverse-peak, the charge of material returned to the aqueous phase is $7.63 \times 10^{-10} \mathrm{C}$, which corresponds to a quantity of $7.9 \times 10^{-15}$ moles or $9.2 \%$ of the material transferred during the forward scan. This indicates that most of the transferred material remained in the organic phase; however no evidence for blockages of the nano-interfaces was seen by performing repeated $\mathrm{CV}$ experiments.

In the case of interfaces formed at the smaller nanopores (design 4 and 5), the CVs were also influenced by the number of cycles applied. When five consecutive CVs were executed, the curves showed a progressive deformation, with the wave for transfer of $\mathrm{TEA}^{+}$declining and tending to merge with the rising background current, together with the peak on reverse scan stretching toward lower potentials (second and third cycles, dashed and dotted lines in Figure 4 B). This evolution is reversible, as the new CV recorded after 15 minutes of rest, at open circuit potential, was identical to the initial cycle.

\section{Influence of the concentration on the limiting currents}

In theory, depending on whether the nanopores are filled with the organic or the aqueous phase, the liquid | liquid interface can be likened to an inlaid or a recessed disk electrode for the transfer of ions from the aqueous phase to the organic phase. Depending on whether the interface is inlaid or recessed, the current at a single interface for an ion $\mathrm{j}$ transferring from the aqueous to the organic phase can be calculated according to the formulas ${ }^{39,40}$ : 


$$
\left|I_{\lim }\right|=\left|4 z_{j} F D_{j} C_{j} r_{a}\right|
$$

or

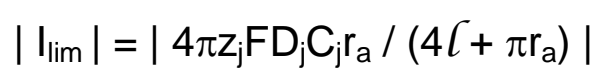

in which $\mathrm{l}_{\text {lim }}$ is the limiting current, $\mathrm{F}$ is the Faraday constant, $\mathrm{r}_{\mathrm{a}}$ is the interfacial radius, $C$ is the length of the pore (the membrane thickness), and $z_{j}, D_{j}$ and $C_{j}$ are, respectively, the charge, the diffusion coefficient and the bulk concentration of the transferred ion j. Equation (1) is used in the case of an inlaid interface configuration and equation (2) in the case of a recessed interface. Equation (1) differs from equation (2) by the factor $(4 / / \pi r)+1$. As a result, equation (2) becomes equivalent to equation (1) when $C$ is zero. The limiting current calculated for one pore must be multiplied by the number of pores $N_{p}$ in order to obtain the total current for the array. As can be seen from these formulas, for an inlaid interface, the limiting current is a linear function of the concentration of $j$ and of the radius $r_{a}$, but for a recessed interface the limiting current is a linear function of the concentration only. However, for the recessed interface, a linear dependence can be observed if the limiting currents are plotted against the term $\pi \mathrm{r}^{2} /(4\lceil+\pi \mathrm{r})$.

The evolution of the limiting current as a function of the term $\pi r^{2} /(4 \tau+\pi r)$ was studied to establish whether the interfaces were recessed or inlaid, for the five sizes of pore available, and for a concentration of $10^{-4} \mathrm{M}$ TEACl. The curves obtained for recesses of 0,50 and $100 \%$ (i.e. $\ell=0,50$ or $100 \mathrm{~nm}$ ) are presented in Figure 5. The experimental limiting currents were determined from background-subtracted voltammograms, at a potential ca. $200 \mathrm{mV}$ positive of the foot of the iontransfer wave. As can be observed in Figure 5, the best linear fit is obtained with a recess of $0 \%$, with a good $R^{2}$ value of $0.99 . R^{2}$ values decreased as the recess increased, to values of 0.84 and 0.78 for $50 \%$ and $100 \%$ recesses, respectively. Considering these results, we conclude that the interface behaves like an inlaid disk electrode: the pores are filled with the organic phase and so the liquid | liquid interface is situated on the aqueous side of the membrane at the mouths of the 
pores. It can be noted than the slope of the line for an inlaid disk interface (Figure 5) is $6.3 \times 10^{-3} \mathrm{~A}$ $\mathrm{m}^{-1}$, which is half the value predicted by equation (1), $1.2 \times 10^{-2} \mathrm{~A} \mathrm{~m}^{-1}$, using $\mathrm{D}_{\text {TEA }}^{+}=7.9 \times 10^{-6} \mathrm{~cm}^{2}$ $\mathrm{s}^{-1}$ in water ${ }^{41}$. This difference will be discussed below.

Following the same procedure, the evolution of the limiting current as a function of the concentration of aqueous phase $\mathrm{TEA}^{+}$was studied at nano-interface arrays formed by each nanopore array design. The forward scans of the CVs obtained with design 1 after backgroundsubtraction and the corresponding calibration curve are shown in Figure 6. As expected, a linear relationship exists between the limiting current and the concentration of TEA ${ }^{+}$. In accord with previous observations, the limiting currents should then be estimable by using equation (1). The theoretical line for the current versus the concentration was calculated and is presented in Figure 6B (dashed line).

As can be seen, the experimental limiting currents (Figure $6 \mathrm{~B}$, solid line) are only ca. $50 \%$ of the calculated currents (dashed line), as already seen when studying the effect of the pore radius on the limiting currents (see above). This has been observed before in other works and this difference between experimental and theoretical current has been attributed to the overlap of diffusion zones formed at adjacent interfaces so that there is not independent diffusion to each interface in the array. In particular, Godino et al. ${ }^{42}$ demonstrated two factors that influenced the currents measured with nanoelectrode arrays: the electrode-to-electrode separation (equivalent to the pore-to-pore separation in the present work, $r_{c}$ ) and the number of nanoelectrodes (equivalent to the number of nanopores or nanointerfaces in the current work, $N_{p}$ ). They showed that as the ratio $r_{c} / r_{a}$ increased, the extent of overlap between adjacent diffusion zones formed at the nanoelectrodes decreased and that the limiting current per pore tended toward that observed for a single nanoelectrode. However, they observed diffusion zone overlap for an electrode-to-electrode separation ratio $r_{c} / r_{a}$ of 60 , which is greater than the one for which no overlap occurs at microelectrode arrays, 20, and which is also the ratio employed in the current investigation. Moreover, they showed that the average current per nanoelectrode tends to decrease as the 
number of electrodes in the array increased, which leads to a current response lower than that expected from equation (1). This was explained by the non-equivalence of the electrodes in an array: the inner electrodes behaved differently than those on the border of the array (edge effect). This loss of current should theoretically reach a limit when the number of inner electrodes far outnumbers the number of outer electrodes. Due to these phenomena, a nanoelectrode array tends to behave like a microelectrode of corresponding dimension. Compared with what is expected from the theory, the limiting currents are smaller, as if measured with a semi-blocked electrode. The same arguments can be applied to the arrays of nano-interfaces employed in this work. Thus the lower-than-theoretical currents measured experimentally (Figure 6B) are due to overlap of diffusion zones formed at adjacent nano-interfaces.

\section{Analytical sensitivity}

The analytical sensitivity, as defined by the International Union of Pure and Applied Chemistry ${ }^{43}$, is the slope of the plot of the analytical signal versus the concentration of the analyte. In electrochemical measurements, the current can be normalised to the geometric area of the electrified interface so that in this case the sensitivity is the slope of the plot of current density, $\mathrm{J}$, versus the concentration of the analyte ion. This normalisation to the interfacial area enables comparison of responses from interfaces of different length scales and also provides a simple indication of the enhanced flux due to convergent diffusion brought about by interface miniaturisation. As the current density is the ratio of the limiting current $\mathrm{I}_{\lim }$ and the interfacial area, the two following formulas, derived from equation (1), can be used to estimate $\mathrm{J}$ and the analytical sensitivity $\mathrm{S}$ in the case of an ideal spherical diffusion regime:

$$
\begin{aligned}
& J=4 Z_{j} F D_{j} C_{j} / \pi r_{a} \\
& S=4 Z_{j} F D_{j} / \pi r_{a}
\end{aligned}
$$


Consequently, the sensitivity is a linear function of the inverse of the radius $r_{a}$, and so should increase as the radius decreases.

The limiting currents determined from the forward scans of CVs were used to calculate the corresponding current density $\mathrm{J}$. Figure 7A shows the five plots of $\mathrm{J}$ versus the concentration of $\mathrm{TEA}^{+}$obtained with each of the five nano-interface arrays studied. As no current due to the transfer of TEA ${ }^{+}$is expected when the concentration of this ion is zero, the origin was included in each data series and set as the intercept for the linear regression analyses. The analytical sensitivities, the slopes of these linear curves, were plotted versus both the nanopore radius and the inverse of the nanopore radius (Figure 7B and $7 C$ ) employed in formation of the nanointerfaces.

The graphs in Figure 7B and 7C show a clear increase in the sensitivity as the pore size decreases, ranging from $65 \mu \mathrm{A} \mathrm{cm}{ }^{-2} \mu \mathrm{M}^{-1}$ at the largest interface size studied here (design 1 , radius $75 \mathrm{~nm}$ ) to $265 \mu \mathrm{A} \mathrm{cm}{ }^{-2} \mu \mathrm{M}^{-1}$ at the smallest interface size studied here (design $5,17 \mathrm{~nm}$ radius). The value of the sensitivity for the $75 \mathrm{~nm}$ radius interface array is in agreement with the value determined previously using a water-organogel interface array of ca. $70 \mathrm{~nm}$ interface radius $^{28}$. It should be noted that the sensitivities determined from these experiments were lower than the calculated ones (dashed line in Figure 7C). Effectively, the experimental values are ca. $50 \%$ of the calculated ones, as expected since the sensitivities derive directly from the experimental limiting currents. This is, as before, attributed to the non-equivalence of the nanointerfaces and overlap of diffusion zones at adjacent interfaces (see above).

The error bars in Figure 7B and $\mathrm{C}$ were determined from the standard deviations of the slopes of the curves in Figure 7A. Greater variation was observed with the smaller nano-interfaces (design 4 and 5, pore radii of 20 and $17 \mathrm{~nm}$, respectively), as the dispersion of the experimental points is greater (Figure 7A). This could be due to the difficulty in determining the limiting currents from the experimental voltammograms and the indirect means employed to measure them. However, it should be observed that Figure $7 \mathrm{C}$ displays a clear linear relationship between the sensitivity and 
the inverse of the radius $\left(R^{2}=0.95\right)$ despite the variation. This linear behaviour is further confirmation that the diffusion of $\mathrm{TEA}^{+}$from the bulk aqueous phase to the interface phase is a radial process and that the membrane pores are filled with the organic phase.

\section{Conclusions}

Voltammetry of $\mathrm{TEA}^{+}$cation transfer has been performed at the water | liquid $\mathrm{DCH}$ interface using five different arrays of nanointerfaces of varying radius. These nanointerfaces were formed by use of nanopore arrays in silicon nitride membranes. The liquid organic phase was found to fill the nanopores, creating inlaid interfaces with the aqueous phase. However, overlap of diffusion zones formed at adjacent nanointerfaces resulted in lower ion transfer currents than if the diffusion zones were independent of each other. The cyclic voltammograms became more complex (background current rising, association with the supporting electrolyte anion) as the interface size decreased. Despite this, an improvement in the analytical sensitivity (calibration graph slope) of the nanointerface arrays was observed. As noted in previous work and by others, ${ }^{26,42}$ nanointerface array behaviour is different than expected from studies with microinterface arrays and the gain in current density and sensitivity is not strictly described by the models which apply at the microscale. Besides the sensitivity improvements reported here, the eventual gain in detection limits that may be achieved with these nanointerface array detection systems remains part of ongoing studies.

\section{Acknowledgements}

This work was supported by Curtin University and the Western Australian Nanochemistry Research Institute (WANRI), a WA State Centre of Excellence. The authors are grateful to Tyndall National Institute, Cork, Ireland, for the gift of the nanopore array membranes. The Curtin 
State and Australian Commonwealth governments. The Nanochemistry Research Institute's

Scanning Probe Microscopy facility was supported by the Australian Research Council.

\section{References}

1. R. A. W. Dryfe, Adv. Chem. Phys., 2009, 141, 153.

2. H. H. Girault, in Electoanalytical Chemistry: A series of Advances, eds. A. J. Bard and C. G. Zoski, 2010, vol. 23, p.1.

3. A. Berduque, M. D. Scanlon, C. J. Collins and D. W. M. Arrigan, Langmuir, 2007, 23, 7356.

4. S. Liu, Q. Li and Y. Shao, Chem. Soc. Rev., 2011, 40, 2236-2253.

5. R. G. Compton, G. G. Wildgoose, N. V. Rees, I. Streeter and R. Baron, Chem. Phys. Lett., 2008, 459, 1-17.

6. R. W. Murray, Chem. Rev., 2008, 108, 2688-2720.

7. Y. Shao and M. V. Mirkin, J. Am. Chem. Soc., 1997, 119, 8103-8104.

8. Q. Li, S. Xie, Z. Liang, X. Meng, S. Liu, H. H. Girault and Y. Shao, Angew. Chem., Int. Ed., 2009, 48, 8010-8013.

9. F. O. Laforge, P. Sun and M. V. Mirkin, J. Am. Chem. Soc., 2006, 128, 15019-15025.

10. P. Sun, F. O. Laforge and M. V. Mirkin, J. Am. Chem. Soc., 2007, 129, 12410-12411.

11. P. Sun, F. O. Laforge and M. V. Mirkin, J. Am. Chem. Soc., 2005, 127, 8596-8597.

12. P. Sun, Z. Zhang, Z. Gao and Y. Shao, Angew. Chem., Int. Ed., 2002, 41, 3445-3448.

13. Y. Yuan and Y. Shao, J. Phys. Chem. B, 2002, 106, 7809-7814.

14. D. Zhan, Y. Yuan, Y. Xiao, B. Wu and Y. Shao, Electrochim. Acta, 2002, 47, 4477-4483.

15. Y. Shao, B. Liu and M. V. Mirkin, J. Am. Chem. Soc., 1998, 120, 12700-12701.

16. C. Cai, Y. Tong and M. V. Mirkin, J. Phys. Chem. B, 2004, 108, 17872-17878.

17. F. Li, Y. Chen, P. Sun, M. Zhang, Z. Gao, D. Zhan and Y. Shao, J. Phys. Chem. B, 2004, 108, 3295-3302.

18. C. Cai and M. V. Mirkin, J. Am. Chem. Soc., 2006, 128, 171-179.

19. H. Hu, S. Xie, X. Meng, P. Jing, M. Zhang, L. Shen, Z. Zhu, M. Li, Q. Zhuang and Y. Shao, Anal. Chem., 2006, 78, 7034-7039.

20. P. J. Rodgers, S. Amemiya, Y. Wang and M. V. Mirkin, Anal. Chem., 2010, 82, 84-90.

21. Y. Wang, J. Velmurugan, M. V. Mirkin, P. J. Rodgers, J. Kim and S. Amemiya, Anal. Chem., 2010, 82, 77-83.

22.

23.

24.

Y. Wang, T. Kakiuchi, Y. Yasui and M. V. Mirkin, J. Am. Chem. Soc., 2010, 132, 16945-16952.

R. A. W. Dryfe and B. Kralj, Electrochem. Commun., 1999, 1, 128-130.

B. Kralj and R. A. W. Dryfe, Phys. Chem. Chem. Phys., 2001, 3, 5274-5282.

M. Platt, R. A. W. Dryfe and E. P. L. Roberts, Langmuir, 2003, 19, 8019-8025.

26. M. D. Scanlon, J. Strutwolf, A. Blake, D. Iacopino, A. J. Quinn and D. W. M. Arrigan, Anal. Chem., 2010, 82, 6115-6123.

27. J. S. Ellis, G. Herzog, B. Glynn and D.W.M. Arrigan, ECS Trans., 2011, 35, 29-44.

28. M. D. Scanlon and D. W. M. Arrigan, Electroanalysis, 2011, 23, 1023-1028.

29.

30.

H. J. Lee, P. D. Beattie, B. J. Seddon, M. D. Osborne and H. H. Girault, J. Electroanal. Chem., 1997, 440, 73-82.

J. Strutwolf, M. D. Scanlon and D. W. M. Arrigan, Analyst, 2009, 134, 148-158.

31. S. E. C. Dale and P. R. Unwin, Electrochem. Commun., 2008, 10, 723-726.

32. S. Fletcher and M. D. Horne, Electrochem. Commun., 1999, 1, 502-512.

33. M. D. Pritzkler, J. Electroanal. Chem., 1988, 243, 57-80.

34. M. Zhou and J. Heinze, Electrochim. Acta, 1999, 44, 1733-1748.

35. M. Platt, R. A. W. Dryfe and E. P. L. Roberts, Elecrochim. Acta, 2003, 48, 2027-2046.

36. R. Knake, A. W. Fahmi, S. A. M. Tofail, J. Clohessy, M. Mihov and V. J. Cunnane, Langmuir, 2005, 21, 10011008.

37. H. Katano, H. Tatsumi and M. Senda, Talanta, 2004, 63, 185-193.

38. M. Rimboud, C. Elleouet, F. Quentel and M. L'Her, Electrochim. Acta, 2010, 55, 2513-2517. 
39. Y. Saito, Rev. Polarogr. Jpn, 1968, 15, 177-187.

40. A. M. Bond, D. Luscombe, K. B. Oldham and C. G. Zoski, J. Electroanal. Chem., 1988, 249, 1-14.

41. S. Wilke and T. Zerihun, Electrochim. Acta, 1998, 44, 15-22.

42. N. Godino, X. Borrise, F. X. Munoz, F. J. Del Campo and R. G. Compton, J. Phys. Chem. C, 2009, 113, 1111911125.

43. L. A. Currie, Pure Appl. Chem., 1995, 57, 1699. 
Table 1. Characteristics of the five different nanopores arrays membranes evaluated by SEM. Pore radius and pore-pore separation are values averaged over 30 pores.

\begin{tabular}{|c|c|c|c|}
\hline Design & $\begin{array}{c}\text { Pore radius, } \\
r_{\mathrm{a}} / \mathrm{nm}\end{array}$ & $\begin{array}{c}\text { Pore-to-pore } \\
\text { separation, } \\
r_{\mathrm{c}} / \mathrm{nm}\end{array}$ & $\begin{array}{c}\text { Number } \\
\text { of pores, } \\
N_{p}\end{array}$ \\
\hline 1 & $75 \pm 9$ & $1500 \pm 17$ & 400 \\
\hline 2 & $50 \pm 6$ & $1000 \pm 14$ & 400 \\
\hline 3 & $25 \pm 6$ & $500 \pm 6$ & 400 \\
\hline 4 & $20 \pm 4$ & $400 \pm 6$ & 400 \\
\hline 5 & $17 \pm 5$ & $300 \pm 6$ & 400 \\
\hline
\end{tabular}


Figure legends.

Figure 1: SEM images of part of nanopore arrays design $3(A), 4$ (B) and 5 (C).

Figure 2: AFM images of nanopore array design 5, containing the smallest size of pore. (A) The complete array. (B) A portion of the array.

Figure 3. Cyclic voltammetry of $100 \mu \mathrm{M}$ tetraethylammonium transfer (left) and corresponding background-subtracted forward scan (right) obtained with nanointerface arrays formed with nanopore array design 1 (A, B), 2 (C, D), 3 (E, F), 4 (G, H) and $5(\mathrm{I}, \mathrm{J})$. CVs in A, C and E were obtained at $10 \mathrm{mV} \mathrm{s}^{-1}, \mathrm{CVs}$ in $\mathrm{G}$ and I were obtained at $5 \mathrm{mV} \mathrm{s}^{-1}$.

Figure 4. Cyclic voltammetry of $100 \mu \mathrm{M}$ of $\mathrm{TEA}^{+}$transfer as a function of $(\mathrm{A})$ the switching potential (solid line: switch at $0.4 \mathrm{~V}$; dashed line: switch at $0.45 \mathrm{~V}$; dotted line: switch at $0.5 \mathrm{~V}$ ) or $(B)$ the number of consecutive scans (solid line: first CV, dashed line: second CV, dotted line: third $\mathrm{CV}$ ). The insets in (A) show the cross-overs of the forward (dotted line) and reverse (dashed line) scans obtained for a switching potential of $0.5 \mathrm{~V}$. All voltammograms were recorded for nanointerface arrays formed using nanopore array design 5 , at $5 \mathrm{mV} \mathrm{s}^{-1}$.

Figure 5. Evolution of the experimental limiting currents as a function of the term $\pi r^{2} /(4 \ell+\pi r)$ for recesses of $0(\diamond), 50(\boldsymbol{\nabla})$ and $100 \mathrm{~nm}(\boldsymbol{\Delta})$. All experiments were for transfer of $100 \mu \mathrm{M}$ of TEACl from aqueous phase to organic phase.

Figure 6. (A) Forward scans of the $\mathrm{TEA}^{+}$transfer recorded with a nanointerface array formed with nanopore array design 1, for aqueous phase concentrations of TEA ${ }^{+}$ranging from 20 to $140 \mu \mathrm{M}$, in steps of $20 \mu \mathrm{M}$. Voltammograms are background-subtracted. (B) The corresponding calibration curve of the limiting currents against the TEA ${ }^{+}$concentration. The dashed line represents the theoretical current calculated using equation 1.

Figure 7. (A) Calibration plots of ion transfer current density $\left(J, \mu \mathrm{A} \mathrm{cm}{ }^{-2}\right)$ versus TEA ${ }^{+}$ concentration $(\mu \mathrm{M})$ for each nanointerface array, based on nanopore design: $1(\boldsymbol{\square}), 2(\mathbf{\Lambda}), 3(\mathbf{X})$, $4(\diamond)$ and $5(+)$. The experimental currents were measured for the forward scan. (B) Plot of sensitivity of the nanointerface array $\left(\mu \mathrm{A} \mathrm{cm} \mathrm{cm}^{-2} \mu \mathrm{M}^{-1}\right)$ as a function of the nanopore radius $r_{a}(\mathrm{~nm})$ used to form the nanointerface. (C) Plot of the sensitivity $\left(\mu \mathrm{A} \mathrm{cm}^{-2} \mu \mathrm{M}^{-1}\right)$ determined from experiments as a function of the inverse of the radius $r_{a}$ of the nanopores used to form the nanointerfaces and compared to the theoretical curve (dashed line). The error bars in (B) and (C) were calculated from the standard deviations of the slopes of the calibration curves in $(A)$. 


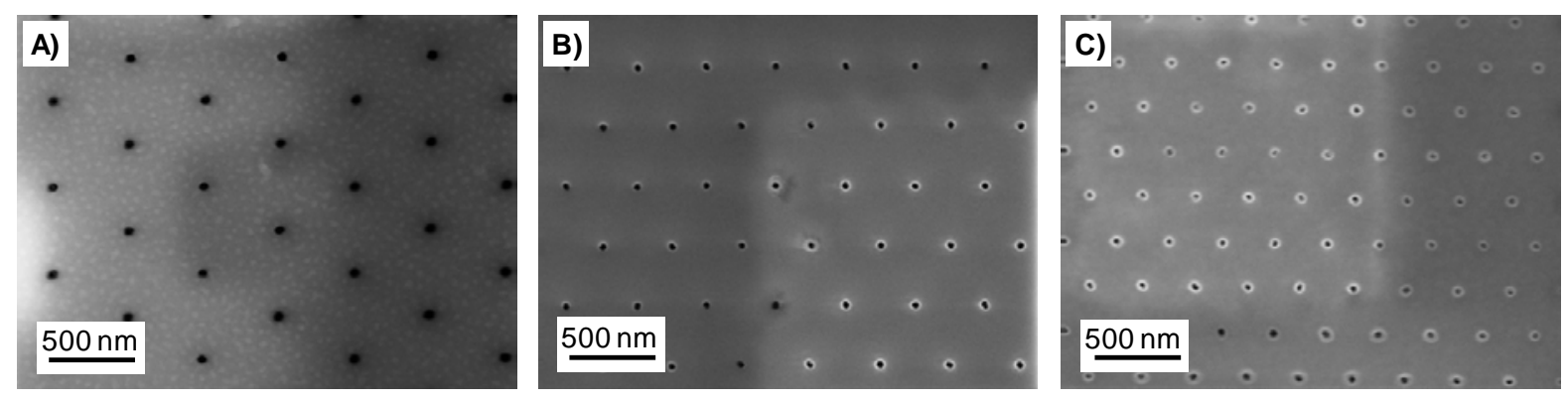

Figure 1. 

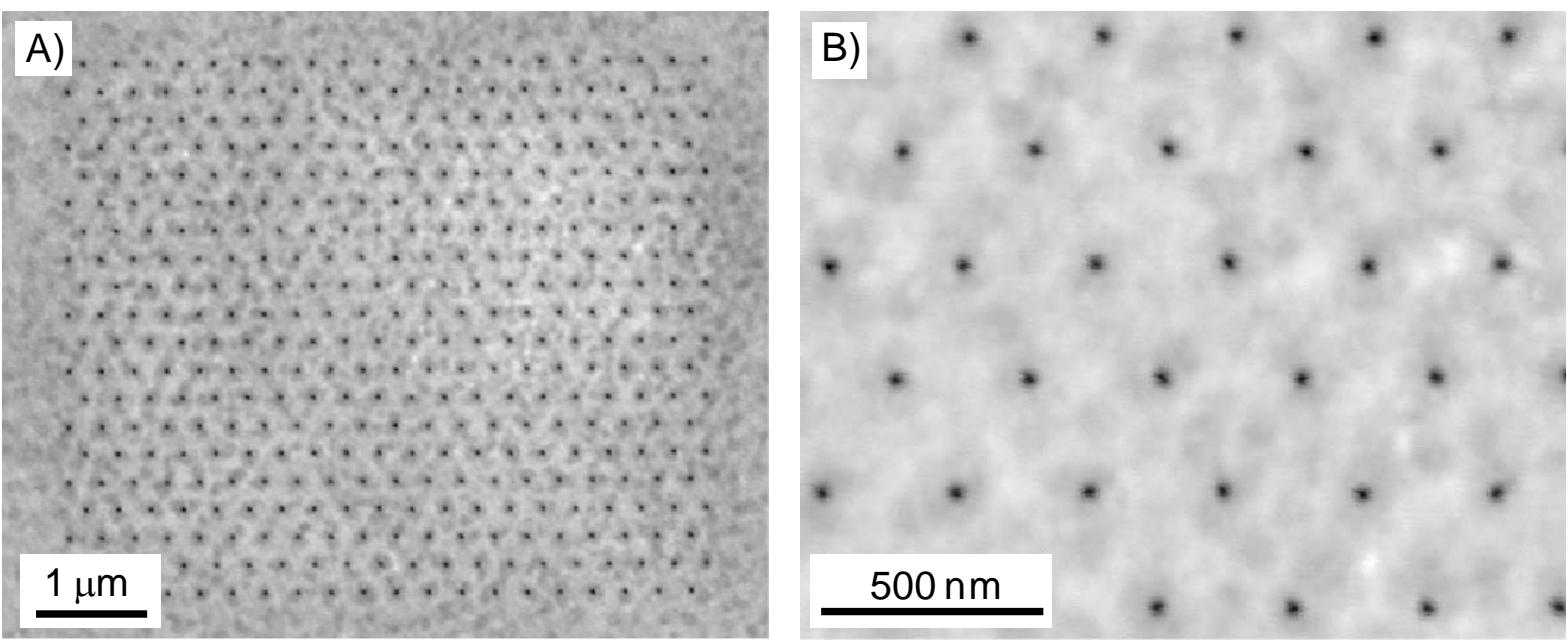

Figure 2. 

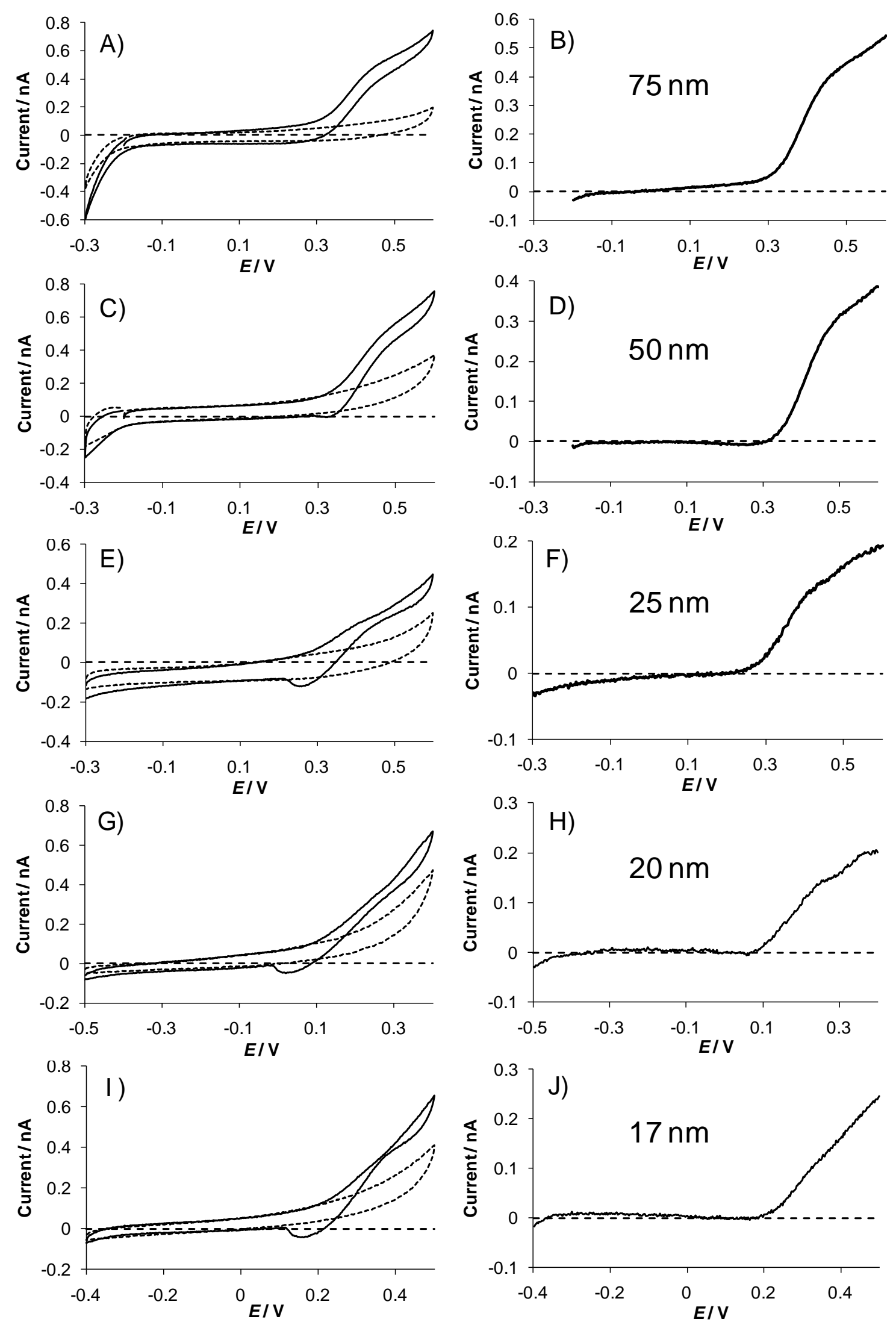

Figure 3. 

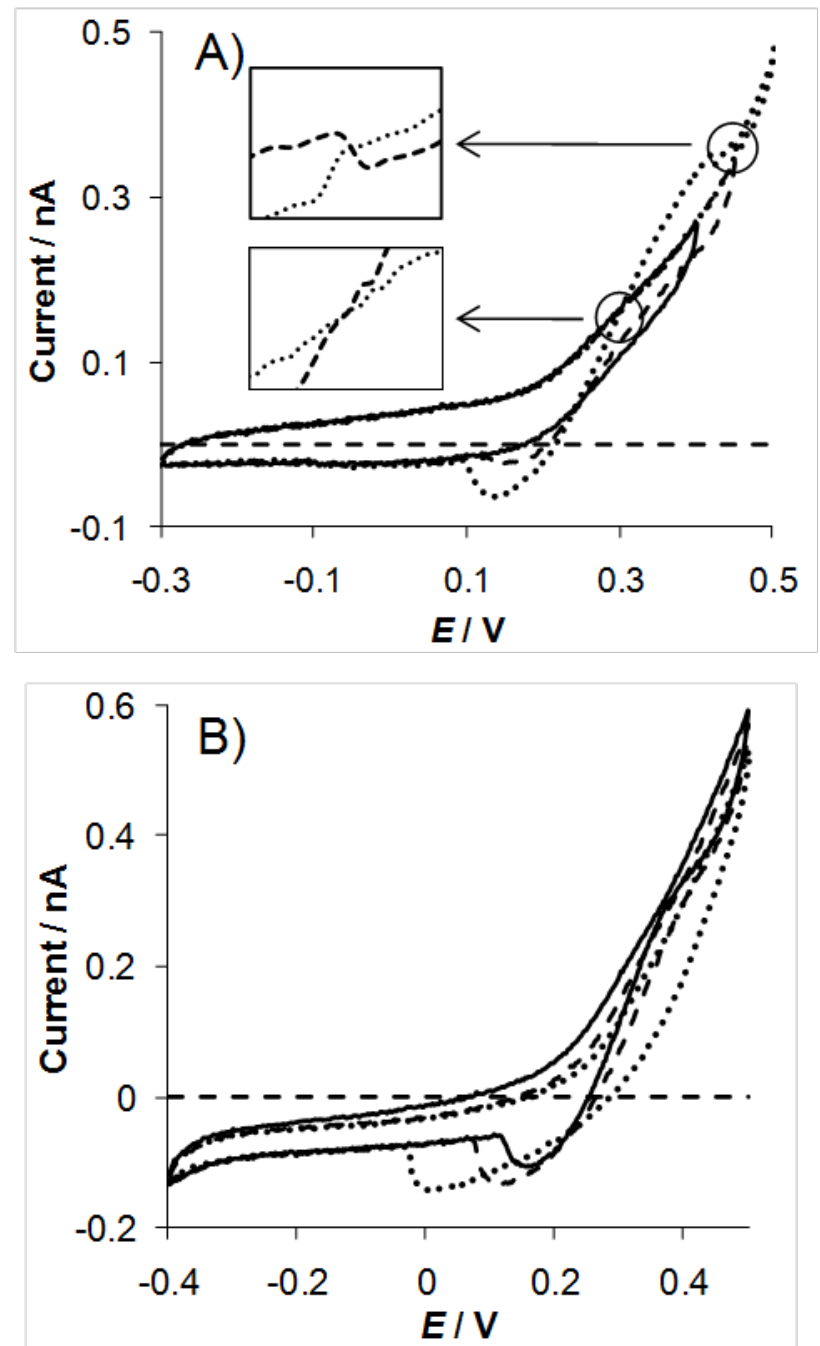

Figure 4. 


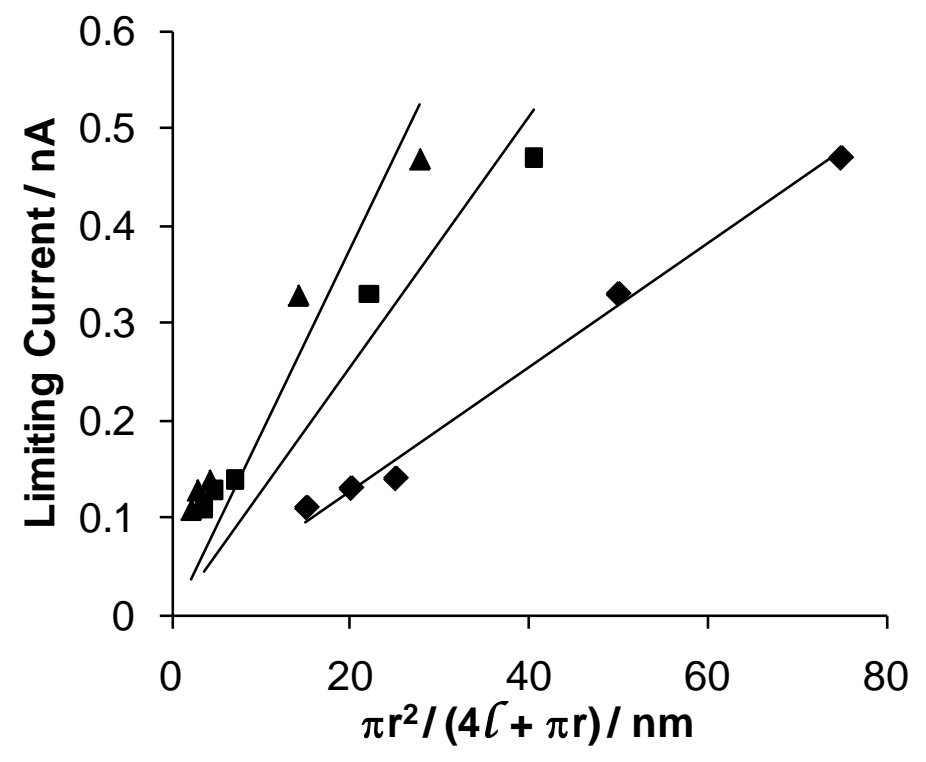

Figure 5. 

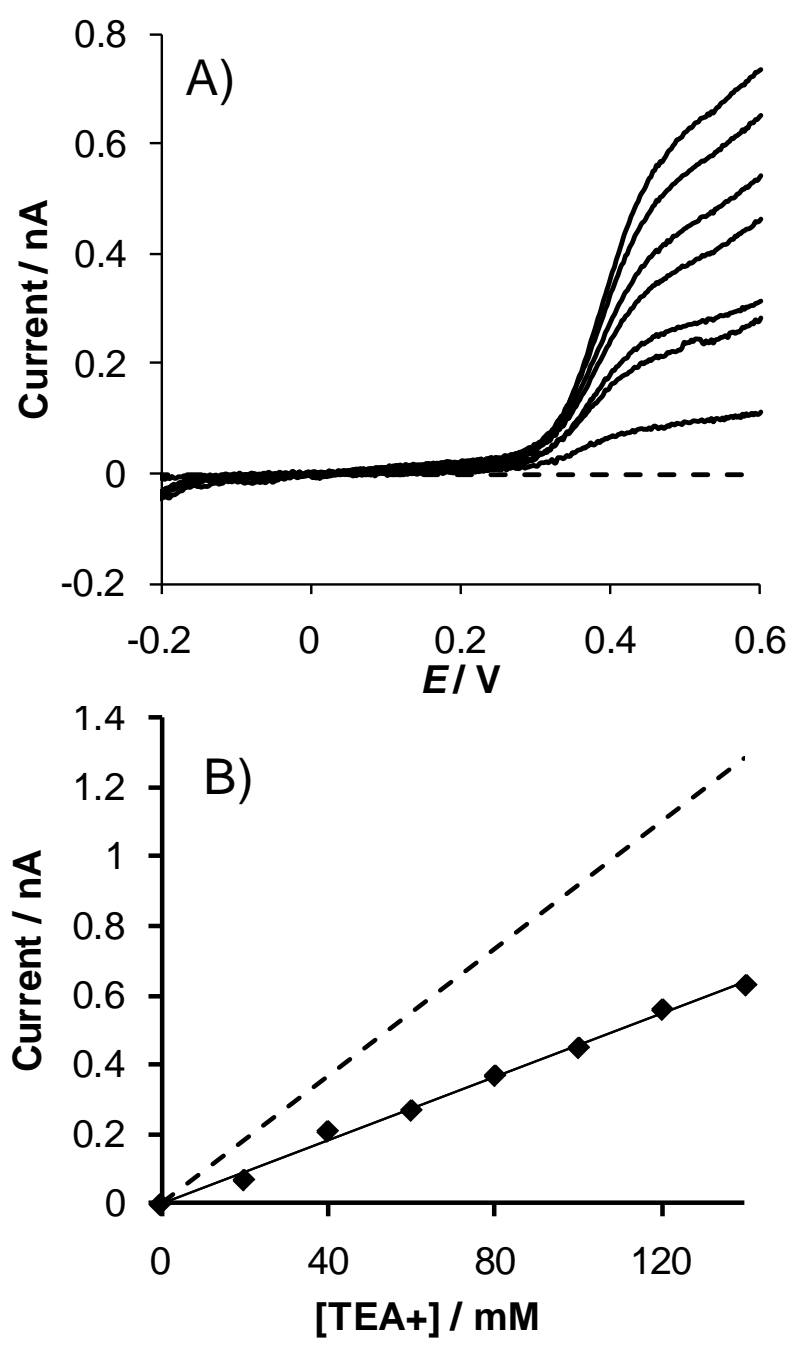

Figure 6. 

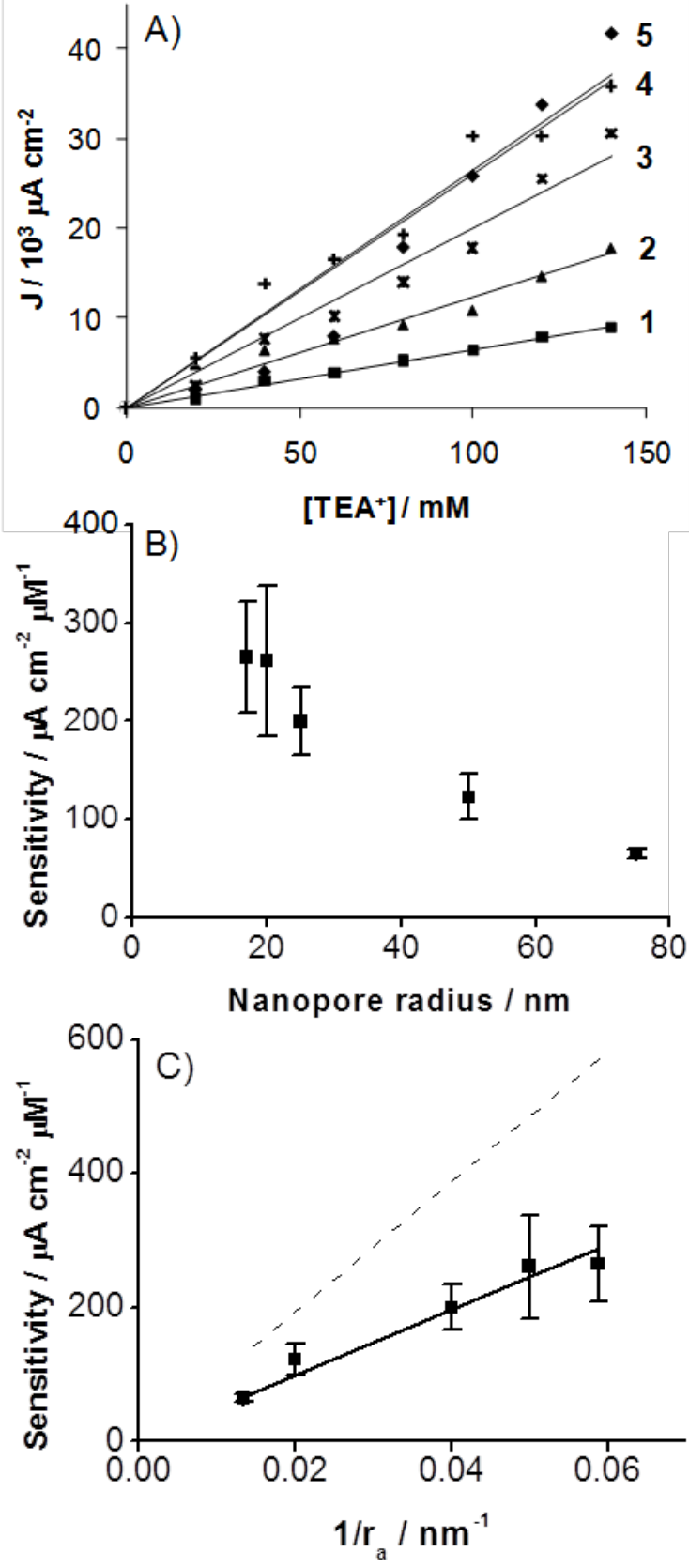

Figure 7. 